\title{
Correction: Amplification of early drought responses caused by volatile cues emitted from neighboring tea plants
}

Jieyang Jin, Mingyue Zhao, Ting Gao, Tingting Jing, Na Zhang, Jingming Wang, Xianchen Zhang, Jin Huang, Wilfried Schwab and Chuankui Song

Correction to: Horticulture Research https://doi.org/10.1038/s41438-021-00704-x, published online 15 November 2021

After the publication of this article ${ }^{1}$, the authors became aware that the word of "tea" was not mentioned in the title. To make the title clear and complete, the word 'tea' is added to the title. The correct version of title is shown below:

Amplification of early drought responses caused by volatile cues emitted from neighboring tea plants
The original article has been corrected.

Published online: 10 December 2021

\section{Reference}

1. Jin, J., Zhao, M. \& Gao, T. et al. Amplification of early drought responses caused by volatile cues emitted from neighboring plants. Hortic. Res. 8, 243 (2021). 